\title{
Situação epidemiológica da tuberculose no Nordeste do estado do Pará, Brasil
}

\section{Epidemiological situation of tuberculosis in the Northeast of the of state of Pará, Brazil}

\author{
DOI: $10.46919 / \operatorname{archv} 1 n 5-\underline{014}$
}

Recebimento dos originais: 10/07/2020

Aceitação para publicação: 30/08/2020

\author{
Nadjane dos Santos Sampaio \\ Graduada em Licenciatura em Ciências Naturais pela Universidade Federal do Pará. \\ Instituição: Instituto de Estudos Costeiros, Universidade Federal do Pará. \\ Endereço: Rua Leandro Ribeiro, s/n. Bairro Aldeia. Bragança PA, Brasil. 68.600.000. \\ E-mail: nadjanesampaio@bol.com.br \\ Aldemir Branco de Oliveira Filho \\ Doutor em Genética e Biologia Molecular pela Universidade Federal do Pará. \\ Instituição: Instituto de Estudos Costeiros, Universidade Federal do Pará. \\ Endereço: Rua Leandro Ribeiro, s/n. Bairro Aldeia. Bragança PA, Brasil. 68.600.000. \\ E-mail: olivfilho@ufpa.br
}

\section{RESUMO}

Este estudo é um levantamento de casos notificados de tuberculose e uma descrição do perfil dos infectados no nordeste do Pará, norte do Brasil. Ele coletou informações dos municípios do nordeste do estado do Pará no Sistema de Informação de Agravos de Notificação (SINAN). Análises de variância dois critérios, Kruskal-Wallis e Qui-Quadrado foram utilizados para comparar os casos de tuberculose em relação a cada microrregião e os fatores de risco. De janeiro de 2003 a dezembro de 2011, 4.900 casos de tuberculose foram notificados no nordeste do Pará. A prevalência e a incidência média anual foi 34,37 e 28,51/100.000 habitantes, respectivamente. Das cinco microrregiões que estão inclusas no nordeste do Pará, a microrregião do Salgado apresentou a maior média anual de incidência e prevalência com 36,64 e 44,70/100.000 habitantes, respectivamente. A microrregião do Guamá apresentou os menores valores das taxas de incidência (20,22/100.000 habitantes) e prevalência $(24,70 / 100.000$ habitantes) médios anuais. As microrregiões do Guamá e Salgado apresentaram um crescimento do número de famílias acompanhadas pela atenção básica com coincidente diminuição dos números de casos de tuberculose. A tuberculose no nordeste do Pará foi mais frequente em indivíduos do sexo masculino $(60,61 \%)$, residentes da zona urbana e proximidades (60\%), na faixa etária de 20 a 39 anos $(45,06 \%)$, com baixa escolaridade $(55,18 \%)$, e predominando em grupos de indivíduos economicamente ativos. A forma clínica mais recorrente foi a forma pulmonar $(94,38 \%)$. Este estudo possibilitou conhecer características da tuberculose no nordeste do Pará, além de avaliar de forma indireta o serviço de saúde destinado ao controle da doença nessa área do Pará.

Palavras-chave: Epidemiologia, Tuberculose, Monitoramento, Pará.

\section{ABSTRACT}

This study is a survey of notified cases of tuberculosis and a description of the profile of those infected in northeastern Pará, northern Brazil. It collected information from the municipalities in the northeast of the state of Pará in the Notifiable Diseases Information System (SINAN). Analyzes of variance two criteria, Kruskal-Wallis and Chi-Square were used to compare the cases of tuberculosis in relation to each micro- 
region and the risk factors. From January 2003 to December 2011, 4,900 cases of tuberculosis were reported in northeastern Pará. The average annual prevalence and incidence was 34.37 and 28.51/100,000 inhabitants, respectively. Of the five microregions that are included in the northeast of Pará, the Salgado microregion had the highest annual mean of incidence and prevalence with 36.64 and 44.70/100,000 inhabitants, respectively. The microregion of Guamá presented the lowest annual average incidence rates (20.22/100,000 inhabitants) and prevalence (24.70/100,000 inhabitants). The micro regions of Guamá and Salgado showed an increase in the number of families accompanied by primary care with a coincident decrease in the number of tuberculosis cases. Tuberculosis in northeastern Pará was more frequent in males $(60.61 \%)$, residents of the urban area and nearby (60\%), aged 20 to 39 years (45.06\%), with low education (55.18\%), and predominating in groups of economically active individuals. The most recurrent clinical form was the pulmonary form $(94.38 \%)$. This study made it possible to know the characteristics of tuberculosis in northeastern Pará, in addition to indirectly assessing the health service designed to control the disease in this area of Pará.

Keywords: Epidemiology, Tuberculosis, Monitoring, Pará.

\section{INTRODUÇÃO}

A tuberculose é uma doença detectada na história da humanidade há milhares de anos ${ }^{1,2}$. Por meio dos avanços tecnológicos, o conhecimento sobre a tuberculose foi ampliado, bem como o desenvolvimento de formas mais eficazes de tratamento da doença, levando as nações desenvolvidas a acreditarem que no fim do século XX, a tuberculose estaria erradicada ou senão confinada a países pouco desenvolvidos. Porém, os intensos movimentos migratórios da população, a falta de estruturados sistemas de saúde pública, a crise social/financeira mundial e o advento da síndrome da imunodeficiência humana (AIDS) tornaram inalcançável esta meta ${ }^{3}$. Atualmente, a tuberculose é um importante problema de saúde pública, em especial em países em desenvolvimento ${ }^{1,2}$.

No Brasil, apesar da tuberculose ser prioridade em muitas ações desenvolvidas pelo Ministério da Saúde e pelas Secretarias de Saúde de Estados e Municípios, ainda são registrados números alarmantes de casos de tuberculose ${ }^{4,5}$. Segundo indicadores do Sistema de Informação de Agravos de Notificação (SINAN/MS), anualmente notificam-se no Brasil em média cerca de 85 mil casos de tuberculose, dentre os quais 70 mil são casos novos. Estima-se ainda que o número de óbitos relacionados à tuberculose seja em torno de 4.600 pessoas no país ao ano ${ }^{4}$. Segundo a Secretaria de Vigilância em Saúde, a tuberculose é a terceira maior responsável pelos óbitos por doenças infecciosas e a primeira entre pacientes com AIDS no Brasil ${ }^{6,7}$.

Pesquisas apontam que ${ }_{2}$ anualmente, o Pará registra em média 3.000 novos casos de tuberculose ${ }^{8}$. Em 2010, a taxa de cura em bacilíferos foi de 73,3\%, sendo que o estabelecido pelo Ministério da Saúde é no mínimo 85\%. Já o abandono do tratamento no mesmo período foi de 8,3\%, quando o indicado pela Organização Mundial da Saúde é inferior a 5\% . A taxa de mortalidade por tuberculose no Pará chegou a superar a taxa nacional de 2,4 contra os 2,7 óbitos por 100 mil habitantes do estado em $2011^{10,11}$. 
A mesorregião do Nordeste do estado do Pará em 2012 representou 15,4\% do número total de casos confirmados de tuberculose com 621 casos confirmados ${ }^{11}$. Desse modo o crescente número de casos de Tuberculose no Pará, mais especificadamente na mesorregião nordeste do Pará, tem se tornado uma realidade cada vez mais preocupante. Além do mais, na mesorregião nordeste paraense ainda são insuficientes os estudos epidemiológicos sobre casos de tuberculose. Sendo assim, torna-se necessário o monitoramento de casos de tuberculose no estado do Pará para melhor direcionar as estratégias e os programas de controle e prevenção de novos casos de tuberculose. Baseado nisso, este trabalho realizou um levantamento de casos notificados de tuberculose e uma descrição do perfil epidemiológico dos infectados nos municípios do nordeste do Pará, norte do Brasil.

\section{MATERIAIS E MÉTODOS}

Segundo o Instituto Brasileiro de Geografia e Estatística (IBGE), a mesorregião nordeste do Pará é composto por 49 municípios agrupados em cinco microrregiões: Salgado, Bragantina, Cametá, Guamá e Tomé-Açu (Figura 1). Ocupando assim, uma área de $83.182,6 \mathrm{~km}^{2}$ que representa $6,7 \%$ da superfície do Estado do Pará12 ${ }^{12}$ A população dessa mesorregião é em torno de 1.789.387 habitantes, o que corresponde a $23,6 \%$ da população do estado do Pará ${ }^{13}$.

Figura 1: Divisão e localização geográfica da mesorregião nordeste do Pará (PA), norte do Brasil. Microrregiões: (1) Cametá; (2) Tomé-Açu; (3) Guamá; (4) Bragantina e (5) Salgado.

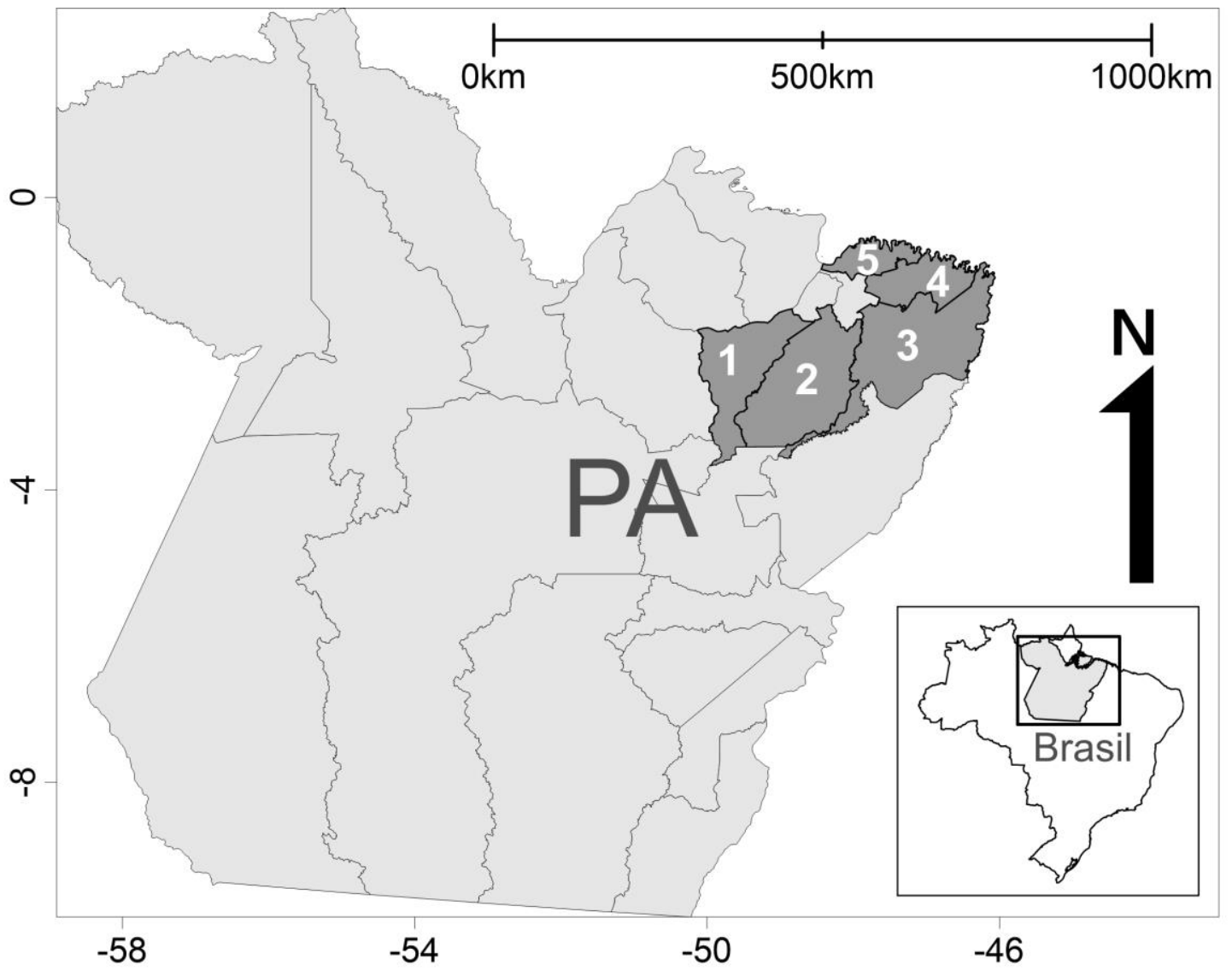


Os dados utilizados neste estudo foram coletados no Sistema de Informação de Agravos de Notificação (SINAN). De acordo com as microrregiões, os números de casos notificados de tuberculose nos municípios da mesorregião nordeste do Pará foram coletados, organizados e analisados. Sendo que, essas informações foram cruzadas com os respectivos dados demográficos (estimativas demográficas baseados no censo 2000, estimativa de 2007 e censo 2010 que foram realizados pelo Instituto Brasileiro de Geografia e Estatística - IBGE) para obter os valores das taxas de incidência e prevalência e a avaliação dos fatores de risco, tais como: sexo, faixa etária, zona de residência, forma clínica e grau de escolaridade. Os dados foram submetidos a testes de normalidade, com nível de significância de 5\%. Para comparar se os casos de tuberculose variaram significativamente em relação à cada microrregião e aos anos em estudos foram realizadas análises de variância dois critérios $(\mathrm{F})$ e, quando violados os pressupostos desse teste estatístico, utilizou-se testes de Kruskal-Wallis (H) e de Qui-Quadrado $\left(\chi^{2}\right)$. Para avaliação e comparações das variáveis que influenciam na frequência dos casos de tuberculose foram utilizados testes de análise de variâncias para as variáveis de grau de escolaridade, forma clínica, zona de residência, sexo e faixa etária. Todas as análises estatísticas foram realizadas utilizando-se o programa BioEstat versão $5.0^{14}$.

Como o presente estudo não abordou diretamente os participantes e utilizou informações de domínio público, não houve necessidade de submetê-lo a avaliação e aprovação por um Comitê de Ética em Pesquisa com Seres Humanos.

\section{RESULTADOS E DISCUSSÃO}

No período de janeiro de 2003 a dezembro de 2011, 4.900 casos de infectados por tuberculose na mesorregião Nordeste do Pará foram notificados. Estimando-se assim uma prevalência e incidência média anual de 34,37 e 28,51/100.000 habitantes, respectivamente. Baseado nessas informações, pode-se sugerir que tais índices estejam associadas aos fatores socioeconômicos da população estudada, tais como precárias condições sanitárias, baixa renda, educação deficiente, aglomerados populacionais, desnutrição e alcoolismo ${ }^{15}$. De acordo com o Programa das Nações Unidas para o Desenvolvimento (2000), cerca de $64,14 \%$ da população dos municípios brasileiros estavam em situação de pobreza, apresentando média de $60,75 \%$ dos habitantes com domicílios tendo abastecimento de água e esgotamento sanitários inadequados ${ }^{16}$. A mesorregião nordeste do Pará pode ser enquadrada nesse cenário. A maioria dos habitantes da mesorregião analisada estão localizados na faixa considerada baixa ou muito baixa e ainda com densidade demográfica muito acima da estadual (4,96 habitantes $\left./ \mathrm{km}^{2}\right)$, com 17,71 habitantes $/ \mathrm{km}^{2}$ $13,16,17$. 
$\mathrm{Na}$ figura 2, diversos picos de incidência de casos de tuberculose podem observados, principalmente nos anos de 2005 (582 casos), 2007 (577 casos) e 2011 (577 casos). Sendo que, detectouse diferença significativa $\left(\chi^{2}=24,12 ; \mathrm{p}<0,01\right)$ entre os anos em estudo. Nota-se também o baixo número de casos nos anos de 2003 e 2010 com 483 e 470 casos confirmados, respectivamente. O baixo número de casos no ano de 2003 seria resultado do fato da tuberculose ter sido considerada uma prioridade a ser combatida pelo governo brasileiro, sendo contemplada nos principais pactos na área da saúde ${ }^{18}$. Dessa forma, possivelmente, uma melhor assistência à tuberculose pelos órgãos de saúde regional e local ocasionou esse número reduzido de notificações de tuberculose na mesorregião nordeste do Pará.

Figura 2. Distribuição anual de casos de infectados por tuberculose em nove anos na mesorregião Nordeste do Pará, Brasil.

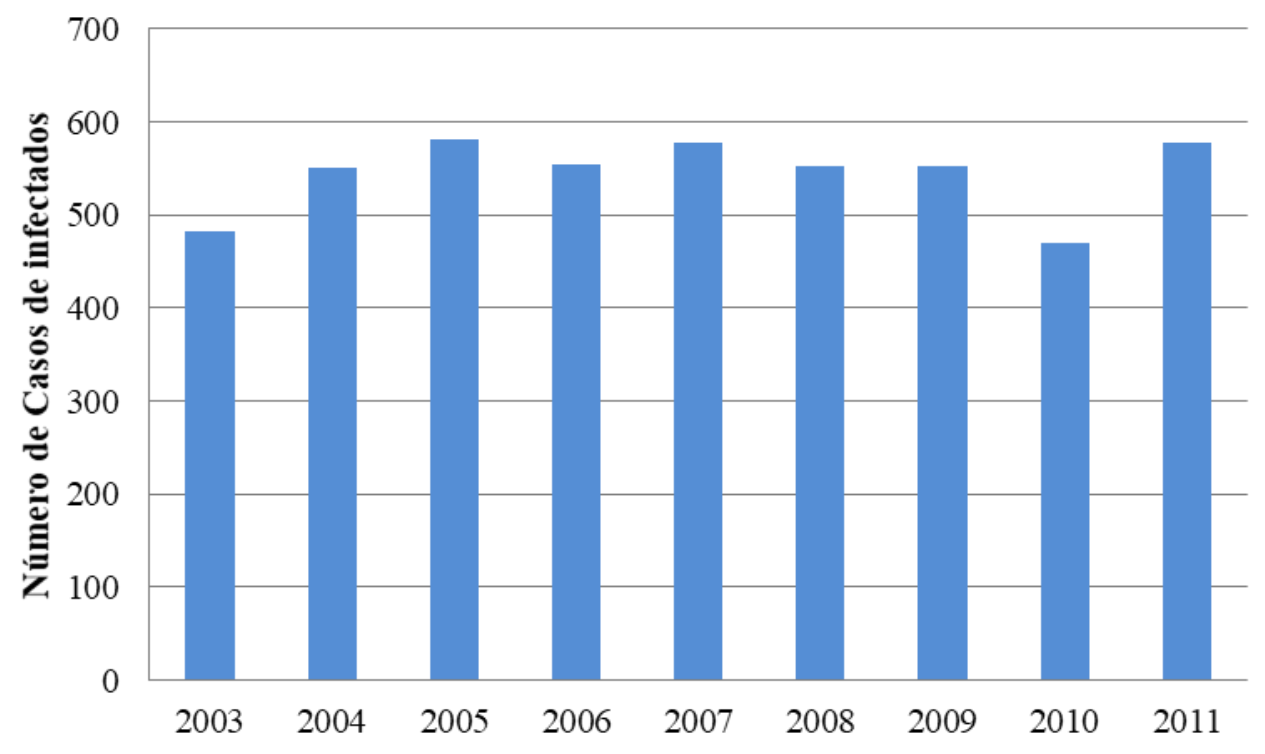

Por outro lado, em 2010, uma considerada redução da população brasileira em situação de miséria foi observada. Em paralelo, uma redução também no número de pessoas com domicílios que dispunham de abastecimento de água e esgotamento sanitários inapropriados (média de 40,76\% no ano de 2010). Essas mudanças se refletiram também no IDH dos municípios da mesorregião nordeste do Pará, onde os novos dados apresentados do ano de 2010 mostraram que 20,4\% dos municípios apresentaram IDH na faixa considerada média e os demais $(79,59 \%)$ permaneceram na faixa de classificação baixa ou muito baixa. Destaca-se que anteriormente estes se apresentavam somente na faixa de classificação baixa ou muito baixa. Isto provavelmente justificaria o baixo número de casos de tuberculose no ano de $2010^{13,16,17}$.

Das cinco microrregiões que estão inclusas na mesorregião nordeste do Pará, a microrregião Bragantina se destacou por apresentar o maior número de casos de infectados por tuberculose, com 1.227 casos notificados, representando $25,04 \%$ do total dos casos registrados na mesorregião nordeste do Pará 
nos nove anos estudados (Figura 3). Porém, foi a microrregião do Salgado que apresentou a maior média anual, concernente as taxas de incidência e prevalência com 36,64 e 44,70/100.000 habitantes, respectivamente. Destacando-se também a microrregião do Guamá com os menores valores das taxas de incidência (20,22/100.000 habitantes) e prevalência (24,70/100.000 habitantes) médios anuais. Estatisticamente, a distribuição dos casos de tuberculose por microrregião apresentou diferença significativa com notável variação de comportamento nas amostras estudadas $(H=22,67 ; \mathrm{p}<0,01)$.

Figura 3: Comparação de serie histórica de casos de tuberculose na mesorregião Nordeste do Pará por microrregião.

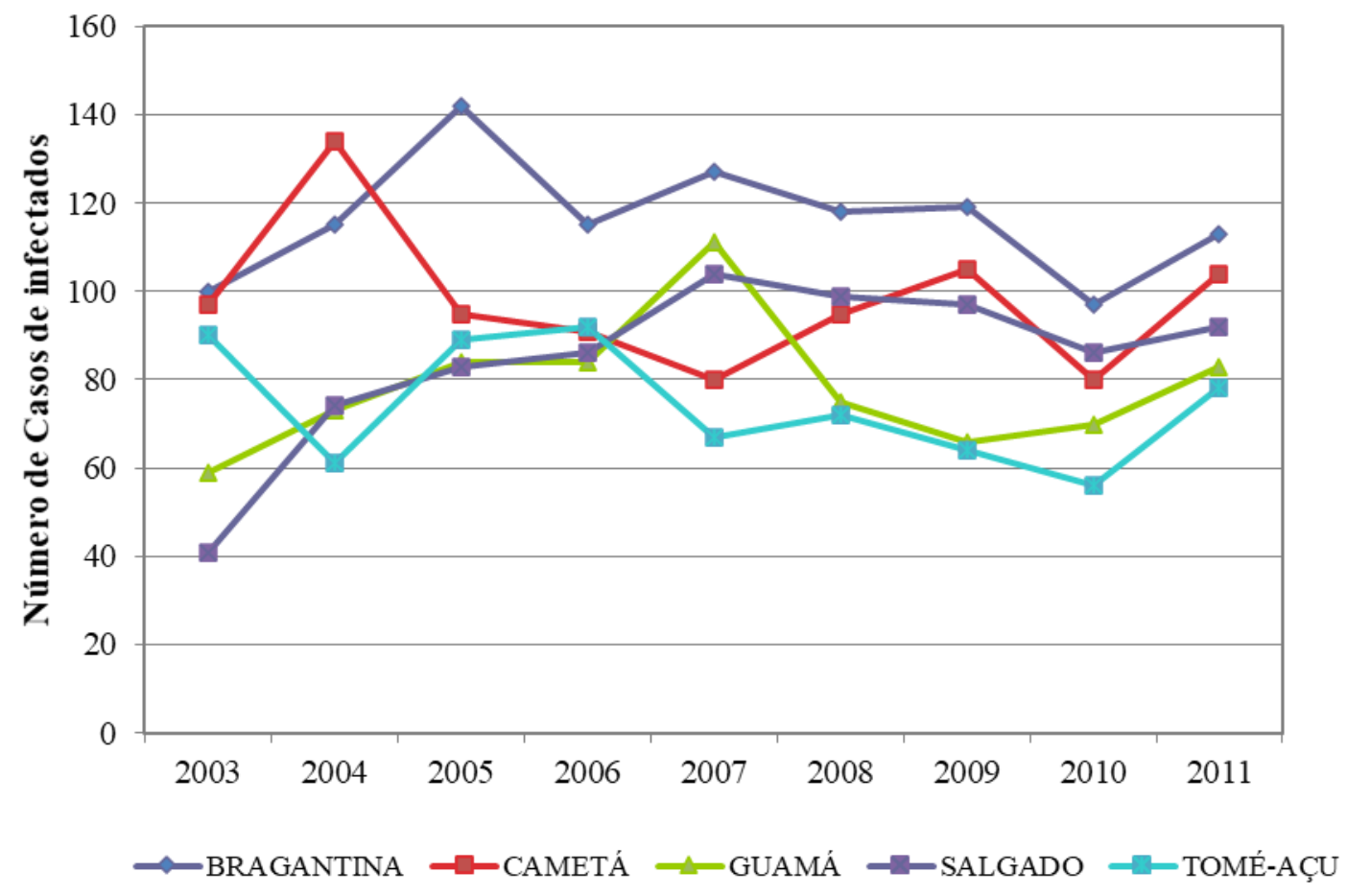

Destacando-se por seus altos índices de notificações de casos incidentes e prevalentes de tuberculose, a microrregião do Salgado Paraense também exibiu altos números referentes aos fatores de risco em detrimento da microrregião do Guamá, que apresentou baixas taxas de incidência e prevalência. A microrregião do Salgado possuía densidade demográfica de valor superiormente considerável se relacionado com da microrregião do Guamá, 37,29 hab./km² (Salgado) e 12,81 hab./km² (Guamá) no ano 2000, diferença que foi mantida no ano de 2010, onde a microrregião do Salgado apresentou 42,71 habitantes $/ \mathrm{km}^{2}$ e a microrregião do Guamá 15,35 habitantes $/ \mathrm{km}^{2}$, sugerindo que a mais alta aglomeração de pessoas seria fator que colabora com a permanência da tuberculose enquanto doença reemergente ${ }^{13,17,19}$.

De acordo com o Programa das Nações Unidas para o Desenvolvimento, as microrregiões do Salgado e Guamá apresentaram declínio no número de pessoas em situação de pobreza, porém essa 
diminuição foi mais expressiva na microrregião do Guamá que no ano 2000 saltou de em média 59,06\% da população em estado de miséria para a média de 28,70\% da população no ano de 2010. Por outro lado, a microrregião do Salgado Paraense que no ano 2000 possuía em média 61,67\% da população em estado de pobreza declinou somente para a média de 47,87\% no ano de $2010^{13,16,17}$. Ao analisar se houve variação significativa na distribuição dos casos de infectados por tuberculose nas amostras durante os anos, comprovou-se estatisticamente que não houve variação significativa na ocorrência destes ao longo dos anos estudados $(\mathrm{F}=0,53 ; \mathrm{p}=0,82)$.

Nas microrregiões do Guamá e Salgado Paraense, microrregiões que apresentaram as maiores e menores taxas de incidência e prevalência nos casos de tuberculose, respectivamente, a relação das famílias acompanhadas pela Atenção Básica está diretamente ligada a melhora na saúde da população assistida, tendo em vista que os números de casos de tuberculose visivelmente diminuíram com o crescimento do número de famílias que são atendidas pelos programas da Atenção Básica de Saúde como se pode verificar na figura 4. Onde se pôde constatar que este fato mais acentuadamente na microrregião do Guamá, portanto as ações promovidas pela atenção básica de saúde contra a tuberculose também tem se mostrado fundamentais para diminuição de casos de infectados ${ }^{20}$.

Dos 4.900 casos notificados na mesorregião Nordeste do Pará, 60,61\% são de pacientes do sexo masculino com o montante de 2.970 casos confirmados. Enquanto que a fração que representada o público feminino que foi afetado pela doença conta com o valor numérico de 1.930 casos notificados. Este achado pode ser explicado por práticas comportamentais mais frequentes no grupo, envolvendo assim ingestão de bebida alcoólica, tabagismo, e o fato do grupo feminino procurar com mais frequência o serviço de saúde, o que acaba por contribuir para o surgimento da doença mais no grupo masculino ${ }^{15,21}$. Tal achado coincide com dados encontrados em várias outras pesquisas na literatura elaborada tanto em nível nacional quanto municipal ${ }^{19,22,23,24}$. 
Figura 4: Comparação entre o número de casos de tuberculose e o número de famílias acompanhadas pela Atenção Básica de Saúde.

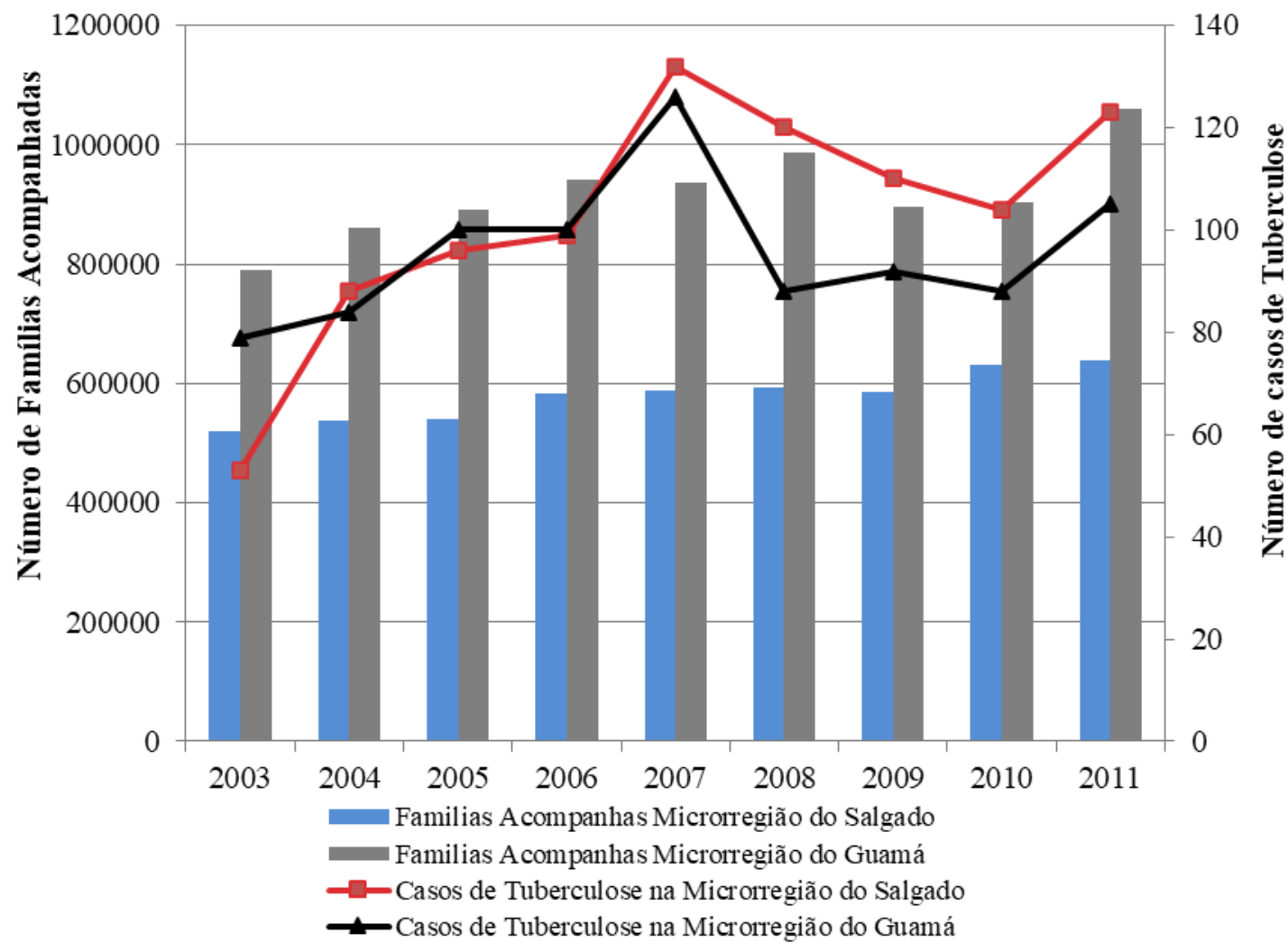

De acordo com o banco de dados disponibilizado pelo SINAN, a maioria (60\%) dos infectados pela tuberculose residem na zona urbana ou em regiões próximas (área periurbana) na mesorregião Nordeste do Pará, com 2.937 casos confirmados (Figura 5). Com diferença estatisticamente significativa da zona rural $(\mathrm{F}=218,96 ; \mathrm{p}<0,01)$.

A urbanização, processo que, muitas vezes ocorre de maneira intensa e desordenada e expõe as pessoas à problemas como o desemprego, violência, precárias condições ambientais, fruto da poluição da água, das péssimas condições sanitárias e de habitação, e a diminuição da área verde que compõe um quadro que muito favorece o acometimento de agravos á saúde como a tuberculose ${ }^{22}$. Este achado de predominância de casos de notificação de tuberculose em indivíduos que residem na zona urbana também foi encontrado em outros estudos ${ }^{15,22}$.

Durante os anos estudados esta diferença se mostrou constante, não havendo estatisticamente variação significativa na distribuição dos casos durante os anos analisados $(F=2,87 ; p=0,078)$. Ressaltase que dos 4.600 casos investigados houve um déficit de 163 casos $(3,3 \%)$ cuja zona de residência dos pacientes não foi identificada. Esta prevalência de casos de tuberculose maior na zona urbana pode ser 
esclarecida pelas altas taxas de urbanização, onde a mesorregião Nordeste apresentou no ano 2000 taxa de urbanização praticamente fixa, dos 47,37\%, com aumento em 2010 para 48,8\% 17,22 .

Figura 5. Série histórica dos casos de tuberculose na mesorregião nordeste do Pará de acordo com a zona de residência.

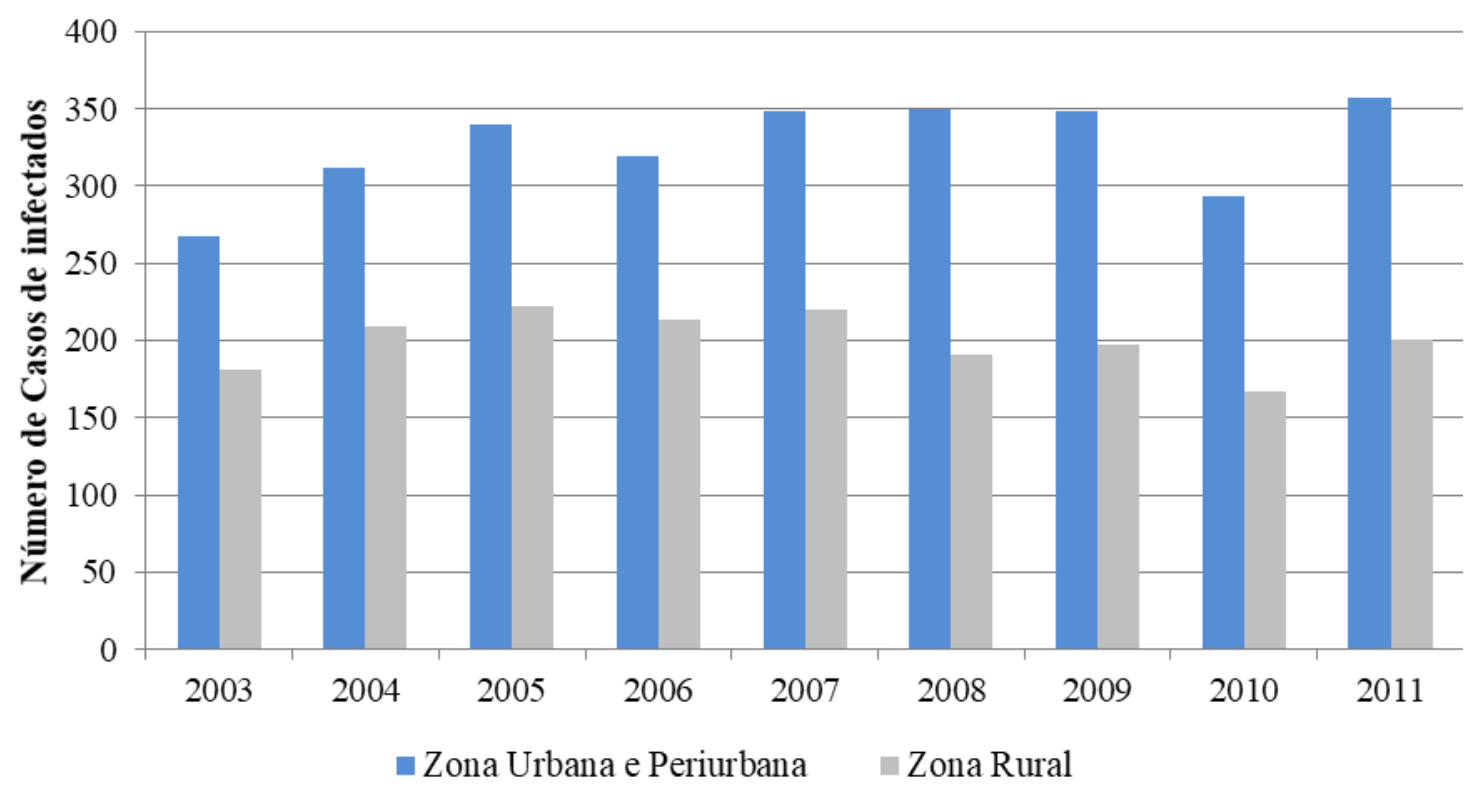

A faixa etária que mais predominou com 2.208 casos confirmados foi a faixa etária dos 20 a 39 anos (Tabela 1), representando 45,06\% dos casos $(\mathrm{F}=463,35 ; \mathrm{p}<0,0001)$. No decorrer dos anos analisados não houve variância significativas na distribuição dos casos. Assim, estatisticamente, observou-se uma constância no número de casos nas faixas etárias examinadas durante os anos estudados $(\mathrm{F}=1,77 ; \mathrm{p}=$ $0,11)$.

O frequente acometimento de indivíduos em idade produtiva (20 a 39 anos) pela tuberculose também foi encontrado em outras pesquisas ${ }^{15,23}$. O recorrente achado faz-nos inferir que a tuberculose ocasiona grandes problemas ao setor econômico, tendo em vista que acarreta prejuízo ao mercado de trabalho e gastos extra à previdência social ${ }^{25}$.

Tabela 1: Número de casos notificados de tuberculose por faixa etária na mesorregião nordeste do Pará.

\begin{tabular}{cc}
\hline Faixa etária & Número de casos $(\%)$ \\
\hline 0 a 9 anos & $70(1,43)$ \\
10 a 19 anos & $522(10,65)$ \\
20 a 39 anos & $2.222(45,35)$ \\
40 a 59 anos & $1.367(27,90)$ \\
60 anos ou mais & $719(14,67)$ \\
\hline Total & $4.900(100,0)$ \\
\hline
\end{tabular}


Observou-se que a maioria dos infectados por tuberculose não possuíam o ensino fundamental completo $(55,18 \%)$, com significativos 2.704 casos de pacientes infectados. Destacou-se também na variável escolaridade como o segundo grupo mais frequente o grupo de infectados descritos como analfabetos representando $10,44 \%$ dos infectados, além do grupo menos frequente representando $0,18 \%$ (com 9 casos confirmados) sendo estes os que possuíam educação superior incompleta $(\mathrm{H}=45,37$; $\mathrm{p}<$ 0,01). Este padrão de frequência em cada modalidade de ensino mostrou-se constante não havendo variância significativa na distribuição dos casos no decorrer dos anos estudados $(\mathrm{H}=1,54 ; \mathrm{p}=0,99)$. Salienta-se que $15,51 \%$ dos casos não se encaixaram em nenhuma das modalidades de ensino, sendo classificada de acordo com o SINAN como "Não se aplica" ou "Ignorados/ Em Branco" (Figura 6). A baixa escolaridade dos infectados muito está associada com os altos índices de abandono do tratamento e óbito por tuberculose, considerando que seu baixo conhecimento com relação às questões de saúde dificulta a compreensão das orientações dadas sobre a tuberculose ${ }^{22,26}$. Resultados semelhantes aos nossos foram encontrado em outros trabalhos ${ }^{19,22,26}$.

A forma clínica da tuberculose mais frequente entre os infectados na mesorregião Nordeste do Pará durante os anos analisados foi a forma pulmonar representando 94,38\% dos casos, com 4.625 casos registrados de infectados. Seguida da forma extrapulmonar representando 4,67\% do valor total, e representando pouco mais de $0,67 \%$ a forma associada da doença, com manifestação da forma pulmonar e extrapulmonar como representado na figura $9(\mathrm{H}=30,97$; $\mathrm{p}<0,01)$. Durante os anos analisados, não foi constatado diferença significativa na distribuição dos casos de tuberculose de acordo com a forma clínica, sendo observado que este padrão prevaleceu durante todos os anos estudados nesta pesquisa $(H=0,72 ; p$ = 0,99). A forma clínica predominante de tuberculose em todos de 2003 a 2011 foi a pulmonar (Figura 7).

Figura 6: Distribuição dos casos de infectados por tuberculose na mesorregião nordeste do Pará de acordo com o nível de escolaridade.

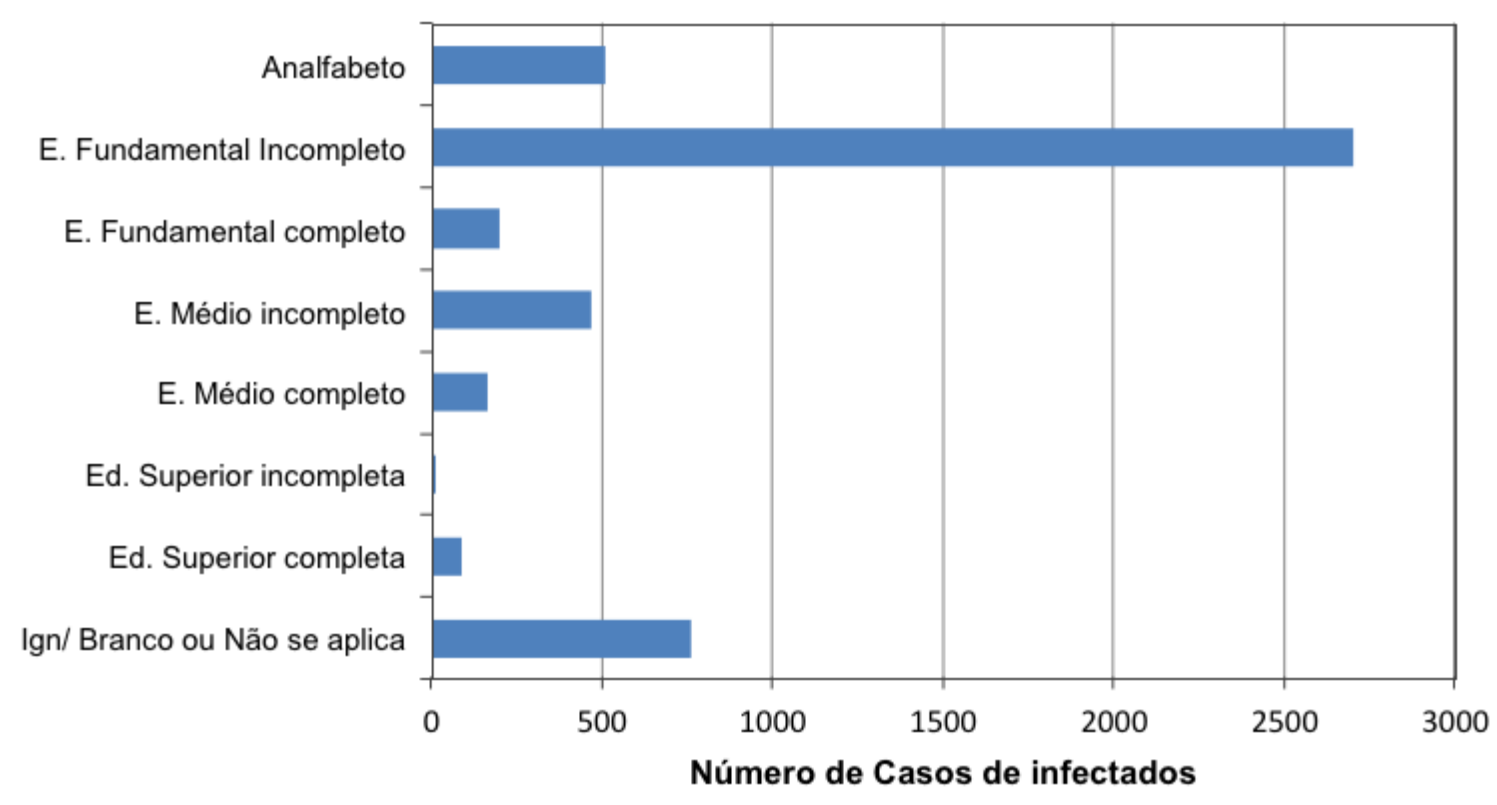


A forma clínica pulmonar, prevalente neste estudo, é de considerável modo um achado preocupante tendo em vista a transmissibilidade, já que na condição de doentes bacilíferos estes, são a principal fonte de transmissão da doença ${ }^{21,27}$. O padrão de frequência da forma clínica pulmonar entre os indivíduos infectados por tuberculose também foi encontrada em outros estudos ${ }^{15,22,23}$.

Figura 7. Distribuição dos casos de infectados por tuberculose de acordo com a forma clínica no decorrer dos nove anos examinados na mesorregião Nordeste do Pará.

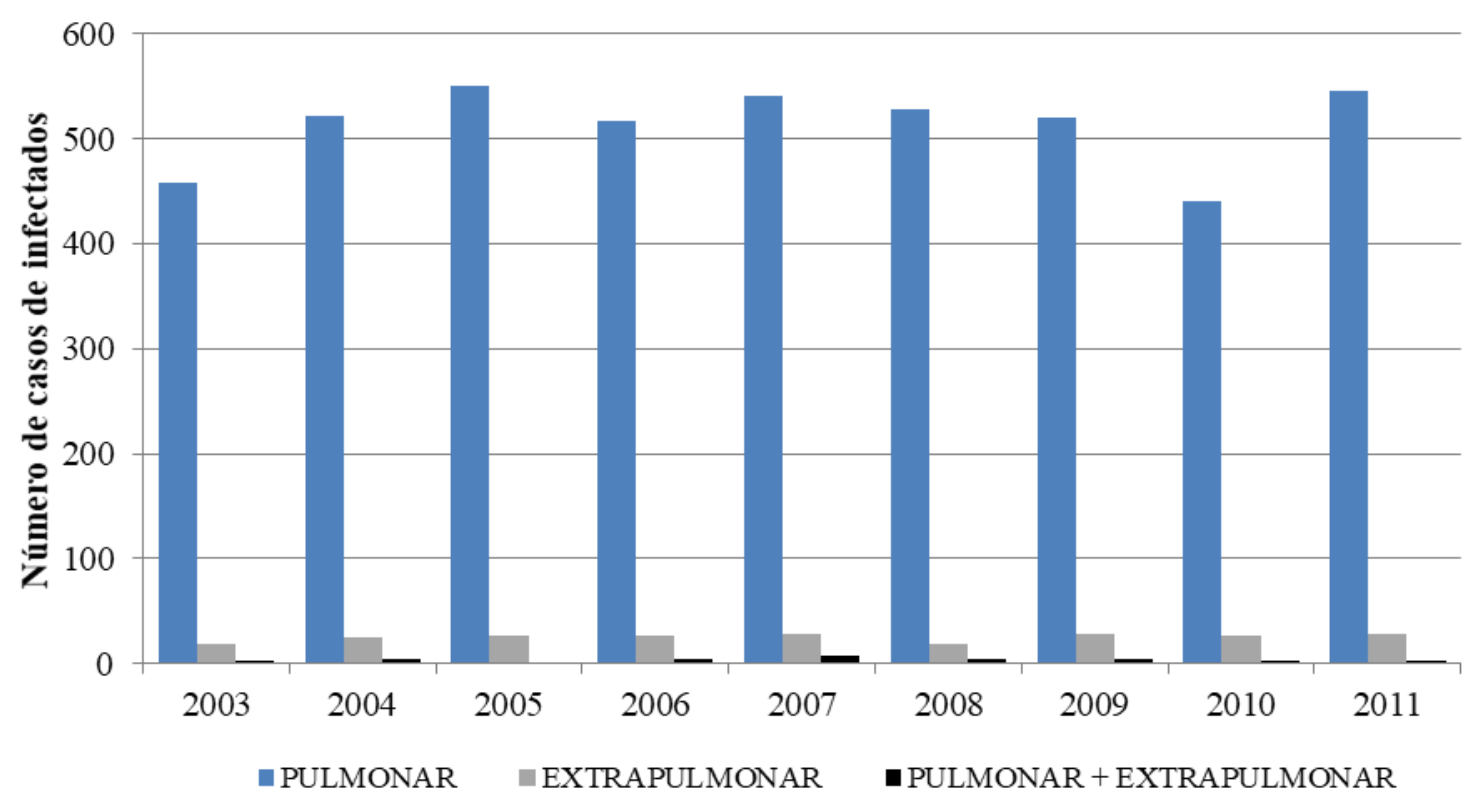

\section{CONCLUSÕES}

Neste estudo verificou-se que no período de janeiro de 2003 a dezembro de 2011 foram notificados na mesorregião Nordeste do Pará 4.900 casos de infectados por tuberculose. Possuindo assim, prevalência e incidência média anual de 34,37 e 28,51/100.000 habitantes, respectivamente.

Das microrregiões que compõem a mesorregião Nordeste do Pará, a microrregião do Salgado apresentou a maior média anual nas taxas de incidência e prevalência com 36,64 e 44,70/100.000 habitantes, respectivamente. Por outro lado, a microrregião do Guamá, destacou-se como a que dispunha dos menores valores das taxas de incidência (20,22/100.000 habitantes) e prevalência (24,70/100.000 habitantes) médios anuais. Observou-se que a tuberculose na mesorregião Nordeste do Pará foi mais frequente em indivíduos do sexo masculino, residentes da zona urbana e proximidades, na faixa etária dos 20 a 39 anos. Com grau de escolaridade baixa (ensino fundamental incompleto), onde a forma clínica mais recorrente foi a forma pulmonar. 
Nas microrregiões do Guamá e Salgado Paraense, a relação das famílias acompanhadas pela Atenção Básica está diretamente ligada a melhora na saúde da população assistida, sendo observado que os números de casos de tuberculose diminuíram com o crescimento do número de famílias que são atendidas pelos programas da Atenção Básica de Saúde. Onde se constatou que este fato mais acentuadamente na microrregião do Guamá, do que na microrregião do Salgado Paraense.

\section{REFERÊNCIAS}

1. Conde MB, Souza GM, Kritski AL. Tuberculose sem medo. Editora Atheneu. $1^{\text {a }}$ ed. São Paulo: 2002.

2. Rosemberg J. Tuberculose - Aspectos históricos, realidades, seu romantismo e transculturação. Bol. Pneumol. Sanit. 1999; 7(2):5-29.

3. Kritski, AL, Conde MB, Souza GRM. Tuberculose do ambulatório a enfermaria. Editora Atheneu. $2^{\mathrm{a}}$ ed. São Paulo: 2000.

4. Secretaria de Vigilância em Saúde, Ministério da Saúde, Brasil. Tuberculose: alinhada com o social, afinada com a tecnologia. Bol. Epidemiol. 2013; 44(2):1-6.

5. Hijjar MA, Oliveira MJPR, Teixeira GGM. A tuberculose no Brasil e no mundo. Bol. Pneumol. Sanit. 2001; 9(2):9-16.

6. Secretaria de Vigilância em Saúde, Ministério da Saúde, Brasil. Tuberculose no Brasil: realidade e perspectivas. Bol Epidemiol. 2012; 43(1):16-9.

7. Jamal LF, Moherdaui F. Tuberculose e infecção pelo HIV no Brasil: magnitude do problema e estratégias para o controle. Rev. Saúde Pública. 2007; 41(Suppl 1):104-110.

8. Waris CDA, Nazaré DL, Marques Júnior JVS, Muniz JWC, Fontelles MJP. Perfil epidemiológico da tuberculose no Hospital Universitário João de Barros Barreto no estado do Pará. Trabalho de Conclusão de Curso, Bacharelado em Fisioterapia, Universidade da Amazônia, Belém, 2007, 73 p. Disponível http://www.unama.br/novoportal/ensino/graduacao/cursos/fisioterapia/attachments/article/135/perfil_e pidemiologico_da_tuberculose_no_hospital_universitario.pdf

9. Neves DCO, Loureiro LO, Paiva NP, Ohnishi MDO, Ribeiro CDT. Análise do Programa de Controle da Tuberculose no estado do Pará, Brasil, de 2005 a 2014. Rev Pan-Amaz Saude. 2018; 9(4):47-56.

10. Lira M. SESPA promove ações para ampliar a prevenção à tuberculose._Agência Pará de Notícias, Diretoria de Comunicação Institucional da Secretaria de Estado de Comunicação, Governo do Pará, 2014. Disponível em: http://www.agenciapara.com.br/noticia.asp?id_ver=119915

11. Ministério da Saúde, Brasil. Sistema de Informação de Agravos de Notificação (SINAN), 2019. Disponível em: http://portalsinan.saude.gov.br/

12. Silva FC, Silva LM. História Regional e Participação Social nas Mesorregiões Paraenses. Paper NAEA 2008: 138. Disponível em: http://www.naea.ufpa.br/naea/novosite/paper/138

13. Instituto Brasileiro de Geografia e Estatística (IBGE). Sistema IBGE de Recuperação Automática SIDRA. $2010 . \quad$ Disponível http://www.sidra.ibge.gov.br/bda/tabela/listabl.asp?z=cd\&o=5\&i=P\&c=608

14. Ayres M, Ayres Junior M, Ayres DL, Santos AAS. dos. BioEstat 5.0 Aplicações estatísticas nas áreasda ciências biológicas e médicas. Belém: Sociedade Civil Mamirauá/CNPq, 2007. 324 p. Disponível em: https://www.mamiraua.org.br/downloads/programas/

15. Coutinho LASA, Oliveira DS, Souza GF, Fernandes Filho GMC, Saraiva MG. Perfil epidemiológico da tuberculose no município de João Pessoa - PB, entre 2007 - 2010. RBCS.;16(1):35-42. 
16. Programa das Nações Unidas para o Desenvolvimento (PNUD). Instituto de Pesquisa Econômica Aplicada (IPEA). Fundação João Pinheiro (FJP). Atlas do Desenvolvimento Humano no Brasil, 2013. Disponível em http://www.atlasbrasil.org.br/2013/pt/o_atlas/o_atlas_/

17. Instituto de Desenvolvimento Econômico, Social e Ambiental do Pará (IDESP). Banco de Dados. Painel de Controle, 2014. Disponível em: http://www.idesp.pa.gov.br/index.php

18. Secretaria de Vigilância em Saúde, Ministério da Saúde, Brasil. Especial Tuberculose. Bol Epidemiol. 2012; 43(1):1-12. Disponível em: http://portal.saude.gov.br/portal/arquivos/pdf/bolepi_v43_especial_tb_correto.pdf

19. Coêlho DMM, Viana RL, Madeira CA, Ferreira LOC, Campelo V. Perfil epidemiológico da tuberculose no Município de Teresina-PI, no período de 1999 a 2005. Epidemiol Serv Saúde. 2010; 19(1):34-43.

20. Secretaria de Vigilância em Saúde, Ministério da Saúde, Brasil. Programa Nacional de Controle da Tuberculose. Plano Estratégico para o Controle da Tuberculose, Brasil 2007-2015. Brasília, 2006. Disponível em: https://www.paho.org/bra/index.php?option=com_docman\&view=download\&category_slug=tubercul ose-971\&alias=927-plano-estrategico-para-o-controle-da-tuberculose-brasil-2007-2015-7\&Itemid=965

21. Monteiro PC, Gazzeta CE. Aspectos epidemiológicos, clínicos e operacionais do controle da tuberculose em um Hospital Escola - 1999 a 2004. Arq Ciênc Saúde. 2007;14(2):99-106.

22. Farias AS, Palha PF. Perfil dos doentes de tuberculose no município de Manaus- Amazonas (2007). Dissertação, Mestrado em Enfermagem em Saúde Pública, Universidade de São Paulo. Ribeirão Preto, 2011, 74p. Disponível em: http://www.teses.usp.br/teses/disponiveis/22/22133/tde-14012011-155730

23. Mascarenhas MDM, Araújo LM, Gomes KRO. Perfil epidemiológico da tuberculose entre casos notificados no Município de Piripiri, Estado do Piauí, Brasil. Epidemiol Serv Saúde. 2005; 14(1): 7-14.

24. Augusto CJ, Carvalho WS, Goncalves AD, Ceccato MGB, Miranda SS. Características da tuberculose no estado de Minas Gerais entre 2002 e 2009. J Bras Pneumol. 2013; 39(3): 357-364.

25. Rosseto DS, Gonçalves PTR. Perfil epidemiológico da tuberculose em Chapecó - SC. Trabalho de Conclusão de curso, Medicina, Universidade Comunitária da Região de Chapecó, Chapecó, 2010. Disponível em: https:/www.unochapeco.edu.br/static/files/trabalhosanais/Pesquisa/Sa\%C3\%BAde/Pablo\%20Tiago\%20Rodrigues\%20Gon\%C3\%A7alves.pdf

Ferreira SMB, Silva AMC, Botelho C. Abandono do tratamento da tuberculose pulmonar em Cuiabá MT - Brasil. J Bras Pneumol. 2005; 31(5):427-435.

Ministério da Saúde, Portugal. Tuberculose: Saiba como controlar, curar e prevenir a tuberculose, uma das doenças que mais pessoas mata, por ano, em todo o mundo. Portal da Saúde, Portugal, 2005. Disponível em: http://www.portaldasaude.pt/portal/conteudos/enciclopedia+da+saude/ministeriosaude/doencas/doencas+i nfecciosas/tuberculose.htm 